\title{
Stimulation of human damaged sperm motility with hydrogen molecule
}

\author{
Kumiko Nakata ${ }^{1,2}$, Naoki Yamashita ${ }^{2}$, Yoshihiro Noda ${ }^{3}$ and Ikuroh Ohsawa ${ }^{1 *}$
}

\begin{abstract}
Background: Sperm motility is a critical factor in male fertility. Low motility can be caused by a variety factors including abnormal spermatogenesis, oxidative damage, or depletion of intracellular ATP. Recent findings indicate that hydrogen molecule $\left(\mathrm{H}_{2}\right)$ selectively reduces toxic reactive oxygen species. In this study, we investigated the effects of $\mathrm{H}_{2}$ on human sperm motility in vitro.

Methods: Experimentally damaged sperm suspensions from patients left at room temperature for $>5$ days or frozen immediately after ejaculation were used. After exposure with $\mathrm{H}_{2}$, their forward motility was measured with a counting chamber. A time-lapse movie was recorded to analyze sperm swimming speed. Mitochondria were stained with a membrane potential-sensitive dye.

Results: $\mathrm{H}_{2}$ treatment significantly improved the rate of forward motility, whereas treatment with nitrogen gas did not. While treatment for 30 min was sufficient to improve motility, it did not affect sperm swimming speed. After $24 \mathrm{~h}$, retreatment with $\mathrm{H}_{2}$ increased the motility again. $\mathrm{H}_{2}$ treatment also increased mitochondrial membrane potential. Forward motility of low motile frozen-thawed sperm from patients significantly improved with cleavage medium containing $\mathrm{H}_{2}$.
\end{abstract}

Conclusions: Our results illustrated that $\mathrm{H}_{2}$ treatment stimulates low sperm motility. $\mathrm{H}_{2}$ is a new promising tool for male infertility treatments.

Keywords: Frozen-thawed sperm, Hydrogen molecule, Male infertility, Mitochondria, Sperm motility

\section{Introduction}

Several factors are present in infertile males with sperm function defects caused by asthenozoospermia [1]. Gene defects, including DNMT3B and MTHFR, have been well documented to correlate with this phenotype [2]. Mitochondria DNA haplogroups may affect sperm motility [3]. Systemic disorders such as polycystic kidney disease [4] also affect fertility and cause asthenozoospermia. Sperms are highly vulnerable to oxidative stress because they contain high concentrations of free unsaturated fatty acids, lack intracellular antioxidant enzymes, and have a limited capacity for DNA repair [5]. The precise mechanisms of motility loss in the sperm, the ability of this cell to fuse with the oocyte under oxidative stress, and the subsequent initiation of lipid peroxidation are not known [6]; however, both oxidative damage to the

\footnotetext{
* Correspondence: iohsawa@tmig.or.jp

${ }^{1}$ Biological Process of Aging, Tokyo Metropolitan Institute of Gerontology,

35-2 Sakae-cho, Itabashi-ku, Tokyo 173-0015, Japan

Full list of author information is available at the end of the article
}

axoneme and depletion of intracellular ATP appear to be involved [7]. While mitochondria are crucial for ATP production, they are also the main source of reactive oxygen species (ROS), notably via the formation of superoxide in the electron transport chain. Nevertheless, low levels of ROS are essential and act as second messengers for the regulation of sperm functions [8].

Previous studies have examined the effects of seminal plasma levels or oral administration of zinc, aspartic acid or coenzyme $\mathrm{Q}_{10}$ on semen quality, and their effects in vitro [9-11]. Especially, myoinositol and xanthine derivates have turned out to be an effective tool in stimulation of sperm motility $[12,13]$. We reported previously that the hydrogen molecule $\left(\mathrm{H}_{2}\right)$ dose-dependently reduces the hydroxyl radical $(\cdot \mathrm{OH})$ in vitro, whereas $\mathrm{H}_{2}$ is too weak to reduce physiologically important ROS such as $\mathrm{NO} \cdot$ and superoxide [14]. $\mathrm{H}_{2}$, the smallest molecule in the universe, has the unique ability of rapidly diffusing across membranes; it can react with cytotoxic $\bullet \mathrm{OH}$ in all 
organelles, including mitochondria and the nucleus, and thus effectively protect cells against oxidative damage. Indeed, $\mathrm{H}_{2}$ prevented a decrease in the cellular levels of ATP synthesized in mitochondria [14]. Many studies reported previously that $\mathrm{H}_{2}$ suppressed oxidative stressinduced injury in several organs, reduced ischemiareperfusion injury in the brain, heart, liver, and retina [14-17], protected against nephrotoxicity [18], and suppressed radiation-induced acute injury in the lung [19]. In the present study, we used experimentally damaged sperm suspensions, and investigated whether $\mathrm{H}_{2}$ treatment exerts protective effects on human sperm. We further demonstrated the practical application of $\mathrm{H}_{2}$ treatment of frozen-thawed sperm from patients.

\section{Methods}

\section{Preparation of sperm suspensions}

Human sperm suspensions from donors were used in this study. This study was approved by the Institutional Review Board of Yamashita Shonan Yume Clinic with consent from patients receiving in vitro fertilization (IVF) treatment at the Yamashita Shonan Yume Clinic. All patients needed IVF and/or intracytoplasmic sperm injection (ICSI) because they showed seminal defects such as hypospermia, oligozoospermia and asthenozoospermia. All procedures followed were in accordance with the ethical standards of the responsible committee on human experimentation (institutional and national) and with the Helsinki Declaration of 1964 and its later amendments. Informed consent was obtained from all patients for being included in the study.

Semen suspensions from patients, who were asked to respect an ejaculatory abstinence period of 3-5 days, were collected by masturbation. A part of them were immediately frozen by the method described below. Sperm parameters were assessed according to World Health Organization criteria (2010) [20]. Collected samples were prepared by washing semen in cleavage medium (SAGE cleavage medium; CooperSurgical, CT, USA) supplemented with $10 \%$ plasma protein fraction (PPF; Baxter Healthcare, IL, USA) to remove seminal plasma, centrifuging through a two-layer Percoll ${ }^{\circ}$ density gradient at $600 \times g$ for $15 \mathrm{~min}$, concentrating by centrifugation at $400 \times g$ for $5 \mathrm{~min}$, and resuspending sperm in cleavage medium with $10 \%$ PPF [21].

\section{Treatment of experimentally damaged sperm with hydrogen molecule}

After IVF using sperm from patients, remaining sperms for discard were used. Each experimentally damaged sperm suspension left in room air at room temperature for $>5$ days was divided into three groups as follows: untreated (i.e., control), $\mathrm{H}_{2}$-treated, and $\mathrm{N}_{2}$-treated. During experiments, sperm suspensions were kept in approximately atmospheric
$\mathrm{O}_{2}$ concentration to enhance oxidative damage. A sperm suspension of $100 \mu \mathrm{L}$ on a culture dish was placed into an exposure chamber (volume, approximately $5 \mathrm{~L}$ ) with $\mathrm{H}_{2}$ mixed gases $\left(5 \% \mathrm{CO}_{2}, 20 \% \mathrm{O}_{2}, 50 \% \mathrm{H}_{2}\right.$, and $25 \% \mathrm{~N}_{2}$ ) or $\mathrm{N}_{2}$-mixed gases $\left(5 \% \mathrm{CO}_{2}, 20 \% \mathrm{O}_{2}\right.$, and $\left.75 \% \mathrm{~N}_{2}\right)$. After closing the exposure chamber tightly, these concentrations of mixed gases $(1 \mathrm{~L} / \mathrm{min}$ flow rate) were reached within approximately $5 \mathrm{~min}$. To confirm saturation of the sperm suspensions with mixed gases, we monitored $\mathrm{H}_{2}$ and $\mathrm{O}_{2}$ concentrations with needle-type sensors (Unisense, Aarhus N, Denmark). After exposure to mixed gases, each sperm suspension was mixed well by pipetting and a $5-10 \mu \mathrm{L}$ drop was placed in a counting chamber [22]. The sperm concentration, forward motility sperm rate, non-forward motility sperm rate, and immobility sperm rate were measured visually three times for each sperm suspension.

To evaluate the velocities of motile sperm, a drop of the suspension was placed in a counting chamber and timelapse movies of sperm movement were recoded using a microscope (Olympus, Tokyo, Japan) for $10 \mathrm{~s}$. Moving images were processed with Image and the CASA (computer assisted sperm analysis) plugin [23]. Forward motility sperm were selected to calculate the velocity.

\section{Treatment of frozen-thawed sperm with hydrogen molecule}

Equal amount of freshly prepared sperm suspension from 21 patients in cleavage medium and TEST-yolk buffer (Irvine Scientific, CA, USA) were mixed and dispended into cryotubes. After exposing to nitrogen steam for 5 minutes, cryotubes were stocked in liquid nitrogen [24]. To thaw frozen sperm solution, cryotubes were warmed at $37^{\circ} \mathrm{C}$ for $5 \mathrm{~min}$, and then each frozen-thawed sperm suspension was dispensed into 4 vials. To prepare the sperm-wash medium containing $\mathrm{H}_{2}$, a 50,75 or $100 \%$ of cleavage medium saturated with $\mathrm{H}_{2}$ was mixed with the medium equilibrated with $5 \% \mathrm{CO}_{2}$. Sperm suspensions were washed for 5 minutes with them, and measured their motility.

\section{Fluorescent staining of sperm mitochondria}

Mitochondria were co-stained with MitoTracker Green (MTG, $2 \mu \mathrm{M}$; Life Technologies, CA, USA) and tetramethylrhodamine methyl ester (TMRM, $2 \mu \mathrm{M}$; Life Technologies) for $30 \mathrm{~min}$. MTG fluorescence was independent of the membrane potential; however, TMRM fluorescence was dependent on the membrane potential. MTG and TMRM were visualized with excitation at 488 and $543 \mathrm{~nm}$, and emission at 510 and $565 \mathrm{~nm}$ with a laser-scanning confocal microscope (Leica, Wetzlar, Germany). Images were analyzed for the membrane potential of individual sperm using TMRM fluorescence intensity values [25]. Sperm viability was assessed by staining with propidium iodine (PI, $10 \mu \mathrm{M}$; Dojindo, 
Kumamoto, Japan) and Hoechst 33342 (10 $\mu \mathrm{M}$; Dojindo). Stained sperm were visualized with excitation at 535 and $350 \mathrm{~nm}$, and emission at 617 and $461 \mathrm{~nm}$ with a laser-scanning confocal microscope, respectively.

\section{Statistical analysis}

All statistical analyses were conducted with JMP (SAS, NC, USA) and essentially performed using one-way ANOVA followed by a post hoc Dunnett's test and twoway ANOVA. Comparison of forward motility before and after treatment of each sperm solution was performed using the paired $t$-test. Differences between data were considered significant for $P$-values $<0.05$.

\section{Results}

Improvement of experimentally damaged sperm motility by treatment with hydrogen molecule

To assess the effect of $\mathrm{H}_{2}$ on sperm motility, we first prepared highly damaged sperms in vitro. Sperm suspensions from patients were left at room temperature for $>5$ days, which enhanced oxidative stress and reduced sperm motility. The average of forward motility rate in 35 suspensions was 3.6\% [90\% confidence interval (CI): 0.6-6.7\%]. We then treated the sperm suspensions with mixed gases containing $50 \% \mathrm{H}_{2}$ gas $\left(\mathrm{H}_{2}\right.$-mixed gases $)$ for $40 \mathrm{~min}$ and found that the forward motility rate increased significantly (16.8\%, 90\% CI: 13.8-19.9\%) (Figure 1a). On the other hand, the rate did not change $(7.7 \%, 90 \% \mathrm{CI}$ : $4.6-10.8 \%)$ after the sperm suspensions were treated with $\mathrm{N}_{2}$-mixed gases (without $\mathrm{H}_{2}$ gas). At that time, $\mathrm{pH}$ was almost equal between suspensions treated with $\mathrm{N}_{2}$ - and $\mathrm{H}_{2}$-mixed gases $(\mathrm{pH} 7.3 \pm 0.1)$. Next, we examined the effect of treatment duration using other suspensions with relatively higher motility $(\mathrm{n}=12)$. Both 30 and 60 min treatments with $\mathrm{H}_{2}$ mixed gas significantly improved sperm motility, whereas the 5 min treatment did not (Figure 1b), indicating that $\mathrm{H}_{2}$ treatment for $30 \mathrm{~min}$ is sufficient to improve motility.

\section{Effect of the sperm survival rate on $\mathrm{H}_{2}$ treatment}

The survival rate of sperm is different in each patient and affects the mobility [26]. We selected other sperm suspensions from 30 patients and examined survival by staining sperm with PI. The average percentage of PInegative, viable sperm in all suspensions was $18.6_{-18.6}^{+35.0} \%$. We then divided them into two groups based on the percentage of viable sperm: high $>20 \%\left(n=17,35.6_{-12.0}^{+20.0} \%\right)$ and low $<20 \%\left(n=13,3.7_{-3.7}^{+9.3} \%\right)$. In low-viability group, sperm were immobile before and after treatment with mixed gases. However, we found that $\mathrm{H}_{2}$ treatment stimulated a very sluggish motility of several sperms with pendular movement of their heads around the axis of forward motility in 3 out of 13 suspensions, indicating that almost immobile sperm remaining a weak ability of movement may be activated by $\mathrm{H}_{2}$ treatment. We further investigated the effects of $\mathrm{H}_{2}$ treatment on sperm in high-viability group, in which the average forward motility was 3.0\% (90.0\% CI: 0.0-10.4\%). After treatment with $\mathrm{H}_{2}$-mixed gas, we found that the forward motility in 16 suspensions significantly increased (Figure 2). Sperm motility in 4 suspensions improved with both $\mathrm{H}_{2}$ - and $\mathrm{N}_{2}$-mixed gases, except for one suspension, which was not activated by both mixed gases. The average forward motilities in sperm suspensions after treatment with $\mathrm{H}_{2-}$ and $\mathrm{N}_{2}$-mixed gases were $11.0 \%$ (90\% CI: $2.7-20.0 \%$ ) and 3.6\% (90\% CI: $0.0-$ $17.6 \%)$, respectively. Thus, $\mathrm{H}_{2}$ treatment effectively and significantly improved sperm motility in suspensions with a higher survival rate.

\section{Effect of $\mathrm{H}_{2}$ treatment on sperm swimming speed}

To examine the effect of $\mathrm{H}_{2}$ treatment on sperm swimming speed, we used time-lapse microscopy and analyzed images with sperm analysis computer software, CASA. Ten sperm suspensions, which were randomly selected from 17 suspensions with a higher survival rate
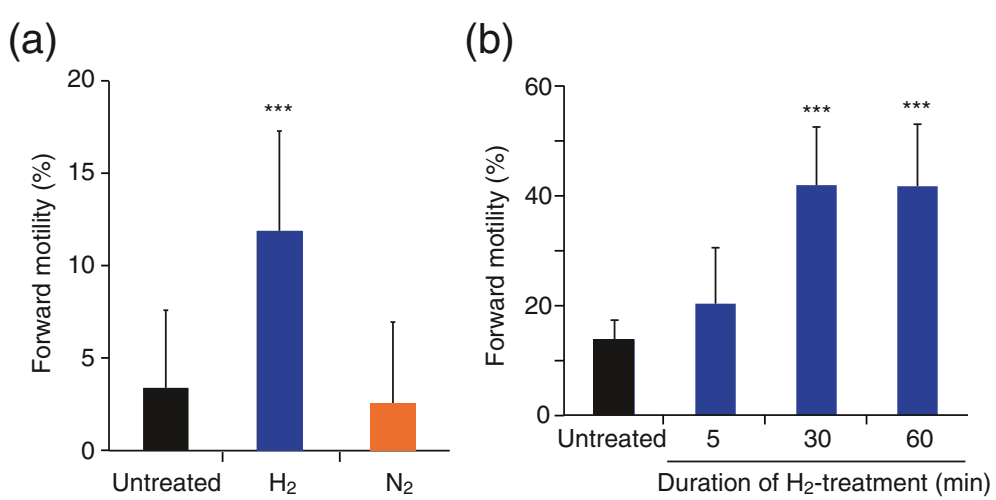

Figure 1 Effect of $\mathbf{H}_{2}$ treatment on human sperm motility. Damaged sperm suspensions $(n=35)$ were untreated or treated with $\mathrm{H}_{2}-$ or $\mathrm{N}_{2}-$ mixed gases for $40 \mathrm{~min}(\mathbf{a})$. Another sperm suspensions $(\mathrm{n}=12)$ were treated with $\mathrm{H}_{2}$-mixed gases for the indicated times $(\mathbf{b})$. Data are represented as means \pm standard deviation (SD). ${ }^{* *} P<0.001$, versus the untreated sperm suspension. 


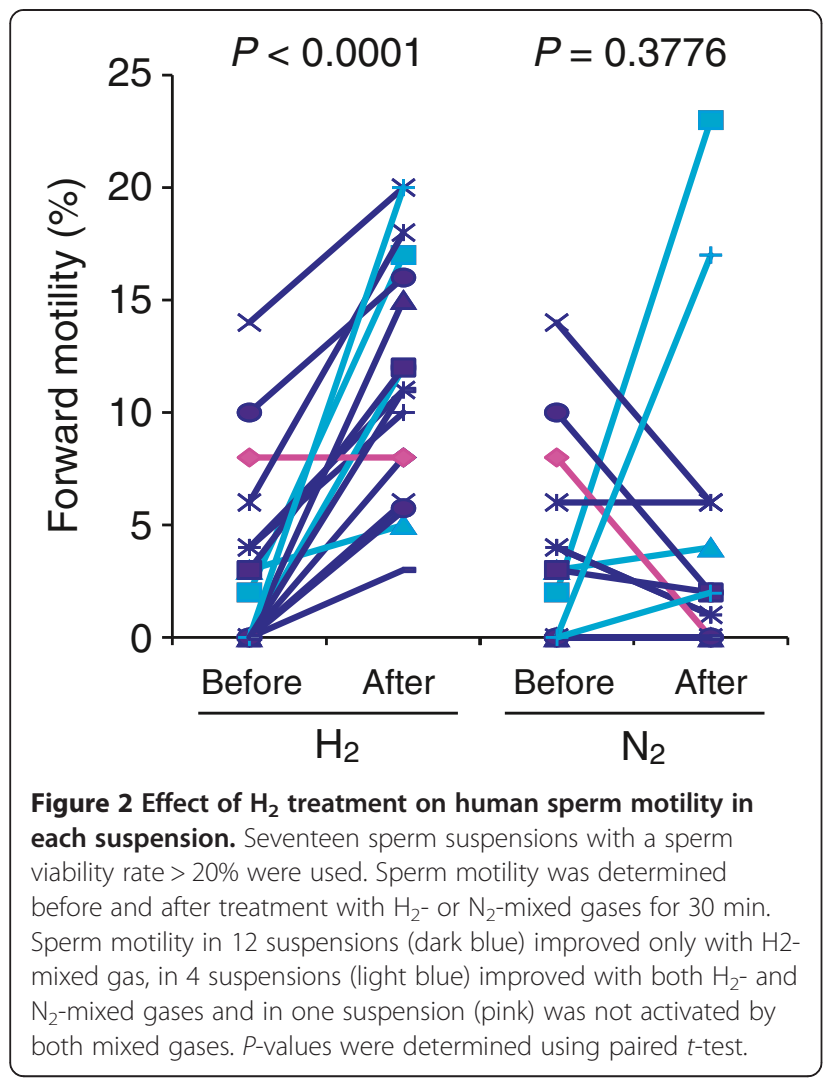

used in Figure 2, were treated with mixed gases for $30 \mathrm{~min}$, and traveling distances of only moving sperm were measured for $10 \mathrm{~s}$ (Figure 3). No significant difference was observed between control and treated groups, indicating that $\mathrm{H}_{2}$ treatment does not affect sperm swimming speed.

\section{Retention time of $\mathrm{H}_{2}$ treatment on sperm motility}

The retention time of motile sperm treated with $\mathrm{H}_{2}$ is an important indicator of IVF and ICSI success. After treatment with $\mathrm{H}_{2}$ - or $\mathrm{N}_{2}$-mixed gases for $30 \mathrm{~min}, 10$ sperm suspensions were kept at $25^{\circ} \mathrm{C}$ in room air. After $2.5 \mathrm{~h}$, we found that forward motility in $\mathrm{H}_{2}$ treated suspension was still higher than that before treatment (Figure 4). It is noteworthy that $\mathrm{H}_{2}$ quickly diffuses. Approximately 10 min after placing an aliquot of the $\mathrm{H}_{2}$-treated sperm suspension in room air, the dissolved $\mathrm{H}_{2}$ concentration reached $<0.1 \%$ of its saturated concentration, indicating that the higher sperm motility enhanced by $\mathrm{H}_{2}$ treatment can be maintained without $\mathrm{H}_{2}$ for at least $2.5 \mathrm{~h}$.

After the first $\mathrm{H}_{2}$ treatment, we further kept a sperm suspension at $25^{\circ} \mathrm{C}$ in room air for $24 \mathrm{~h}$ and found that its motility still remained a level approximately $36 \%$ of that of the first treatment (Figure 4). However, it was not significantly higher than that before treatment. We then treated this sperm suspension with $\mathrm{H}_{2}$ for $30 \mathrm{~min}$ (second treatment) and kept it at $25^{\circ} \mathrm{C}$ in room air for

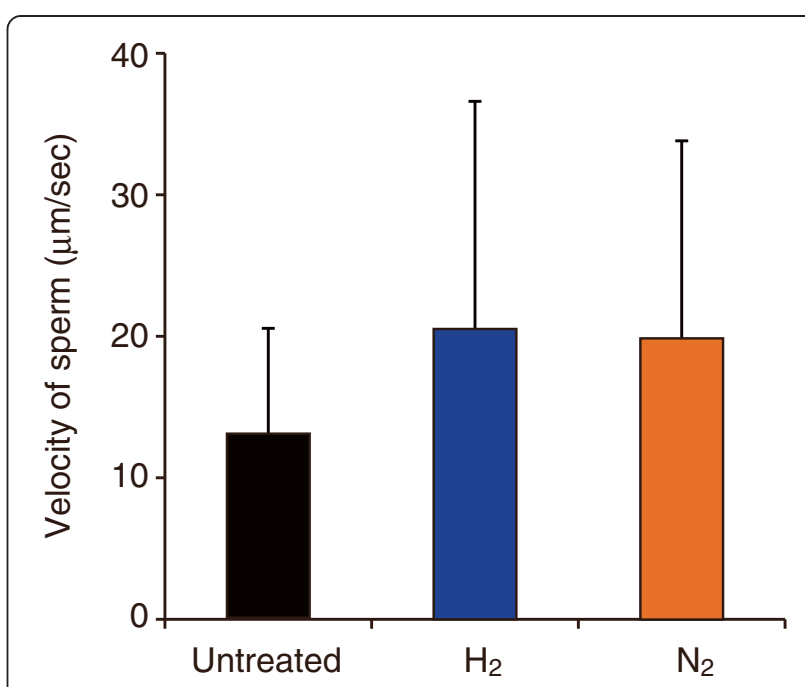

Figure 3 Effect of $\mathrm{H}_{2}$ treatment on human sperm velocity. Ten sperm suspensions were treated with $\mathrm{H}_{2}$ - or $\mathrm{N}_{2}$-mixed gases for $30 \mathrm{~min}$, and time-lapse movies of sperm motility were recorded with a microscope for $10 \mathrm{~s}$. No significant difference was observed between control and treated groups. Data are represented as means \pm standard deviation (SD).

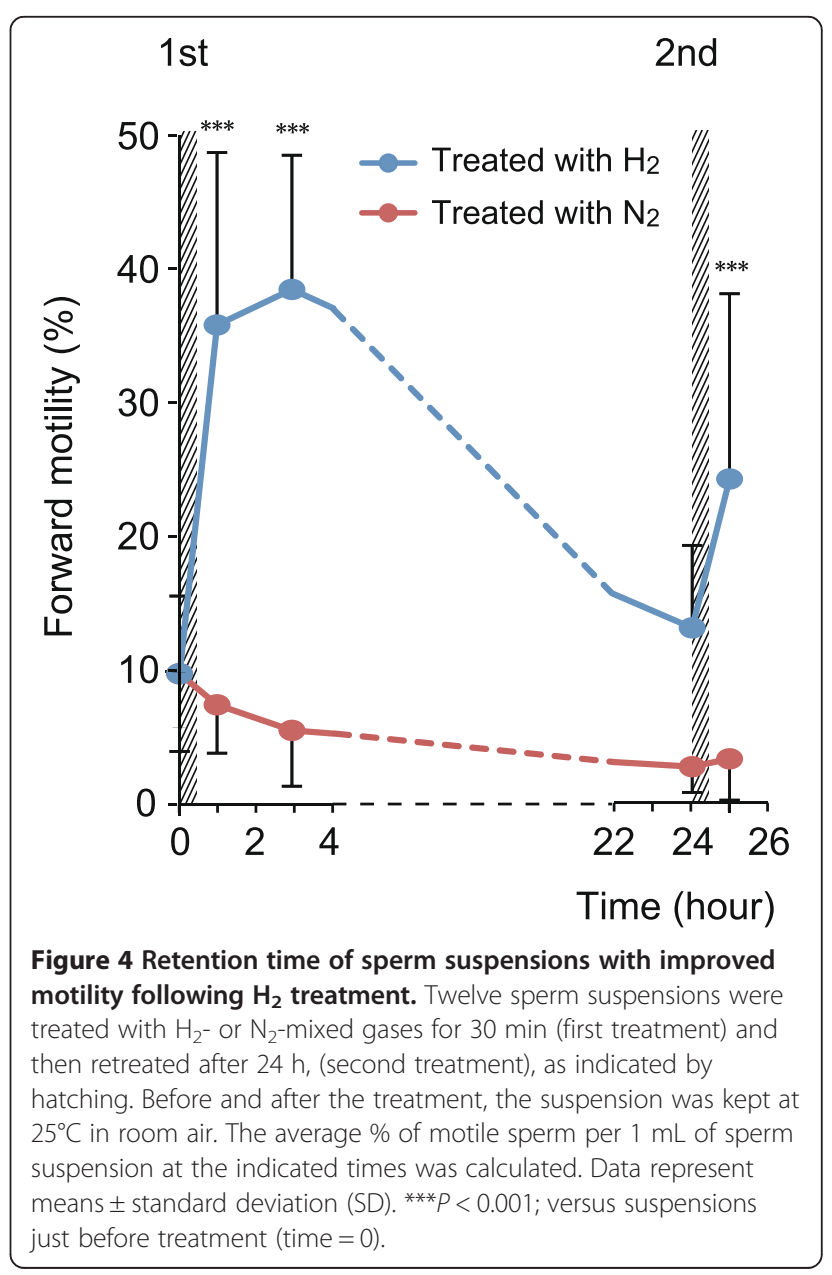


$30 \mathrm{~min}$, and found that sperm motility increased again, reaching a level approximately $67 \%$ of that of the first treatment.

\section{Enhancement of mitochondrial membrane potential by $\mathrm{H}_{2}$ treatment}

Since sperm motility is dependent on ATP content, we hypothesized that the improvement of sperm motility by $\mathrm{H}_{2}$ treatment might be due to changes in mitochondrial function. Therefore, after treatment with $\mathrm{H}_{2^{-}}$or $\mathrm{N}_{2^{-}}$ mixed gases for $30 \mathrm{~min}$ by the same method described in Figures 1, 2 and 3, we stained sperm with TMRM, a mitochondrial membrane potential-dependent dye, and found that TMRM fluorescence increased (Figure 5), suggesting that $\mathrm{H}_{2}$ treatment may enhance mitochondrial function and sperm motility.

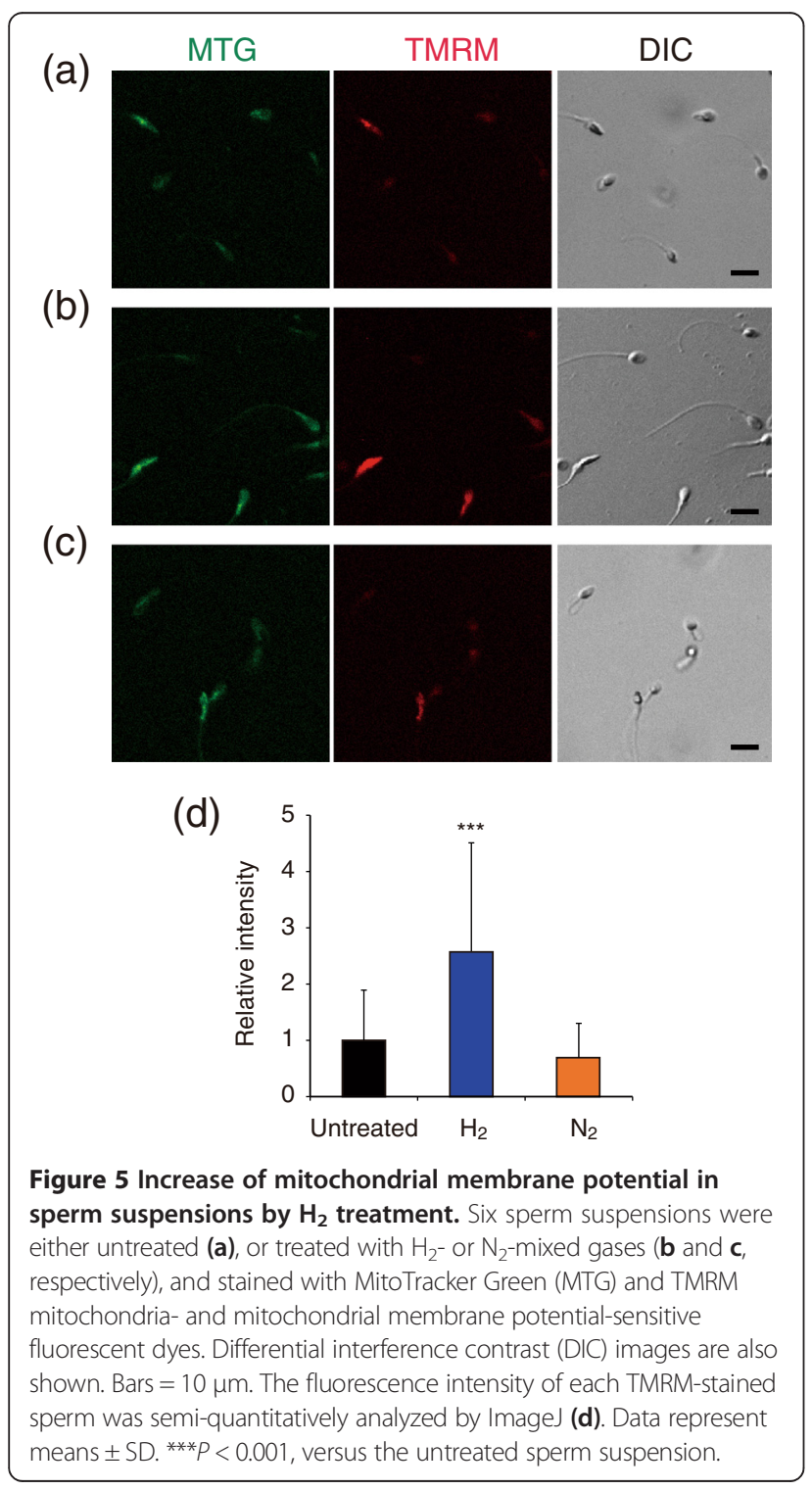

Improvement in frozen-thawed sperm motility by treatment with hydrogen molecule

To demonstrate the practical application of $\mathrm{H}_{2}$, we prepared frozen-thawed sperm suspension from each patient, which could be used for infertility treatment. The average forward motility of total 21 suspensions was 37.1\% (95\% CI: 28.7-45.6). Asthenozoospermia was defined according to the WHO guideline as samples with $<50 \%$ progressive sperm motility [20]. Therefore, we divided them into two groups, higher (forward motility $\geq 50 \%, \mathrm{n}=6$ ) and lower $(<50 \%, \mathrm{n}=15)$ suspensions, of which the average forward motilities before $\mathrm{H}_{2}$-treatment were $61.3 \%$ (95\% CI: 50.8 $71.8 \%$ ) and $27.5 \%$ (95\% CI: $21.7-33.2 \%$ ), respectively. All suspensions were treated with sperm-wash media containing different concentrations (50, 75, and 100\%) of $\mathrm{H}_{2}$. Dunnett's $t$-test for unpaired values indicated that the motility in suspensions of lower motile, asthenozoospermic sperms significantly increased after treatment with sperm-wash media containing $50 \%$ of $\mathrm{H}_{2}$, whereas treatment with $75 \%$ and $100 \%$ of $\mathrm{H}_{2}$ did not (Figure $6 \mathrm{a}$ ). $\mathrm{H}_{2}$ treatment did not show any dose-dependency. The motility both in total and suspensions of higher motile, normozoospermic sperms did not significantly increase after $\mathrm{H}_{2}$-treatment. Next, we compared the forward motility before and after treatment of each sperm suspension by using the paired $t$-test. The forward motility in suspensions of higher motile sperms moderately increased after $\mathrm{H}_{2}$-treatment (Figure 6b). Treatments with $75 \%$ of $\mathrm{H}_{2}$ significantly improved the motility of suspensions, whereas treatments with $50 \%$ and $100 \%$ of $\mathrm{H}_{2}$ did not. On the other hand, we found that the forward motility in suspensions of lower motile sperms apparently increased after treatment with sperm-wash medium containing both $50 \%$ and $75 \%$ of $\mathrm{H}_{2}$ (Figure $6 \mathrm{c}$ ), whereas treatment with $100 \%$ of $\mathrm{H}_{2}$ did not. These results indicated that $\mathrm{H}_{2}$-treatment effectively and significantly improved the motility of frozen-thawed sperms, especially low motile, asthenozoospermic ones.

\section{Discussion}

Since previous studies have shown the balance between ROS and antioxidants to be unequivocally important for a variety of functions in the male reproductive system, we hypothesized that $\mathrm{H}_{2}$ treatment may be protective as a weak scavenger of ROS and may improve sperm motility in vitro. There are two reasons why sperm suspensions left at room temperature for $>5$ days were used. Firstly, ejaculated sperm must be kept for 3 days at our clinic for infertility treatments, and remaining sperms for discard were used. Secondly, $\mathrm{H}_{2}$ is stable and must be coaxed with strong catalysts to enter into chemical reactions, and has been treated as an inactive gas in our body, indicating the possibility that the effects of $\mathrm{H}_{2}$ on sperm are not strong. Then, we expected that the using 


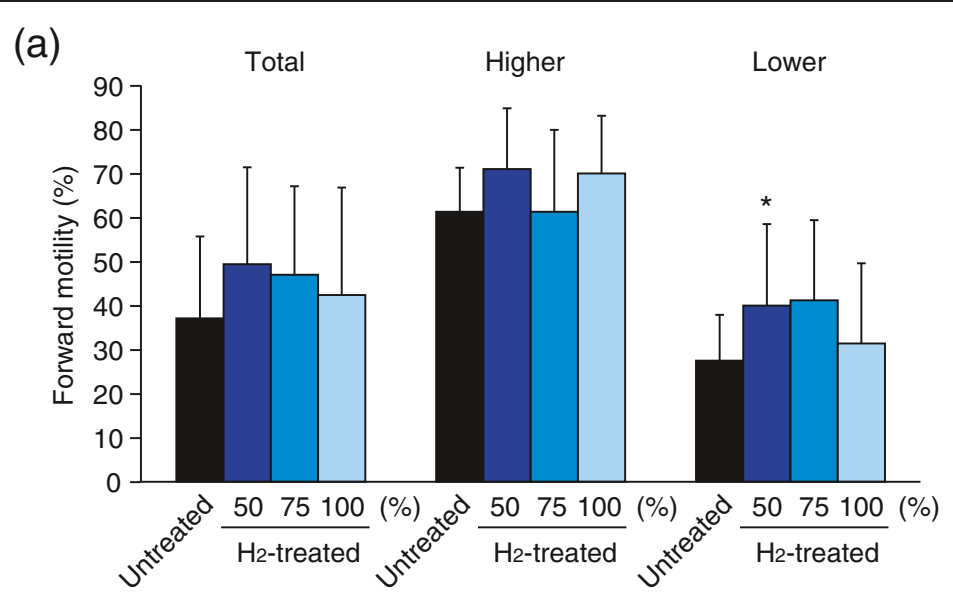

(b)

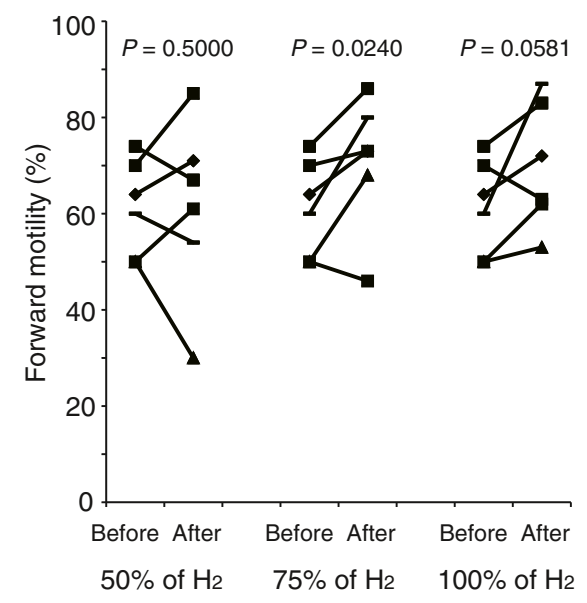

(c)

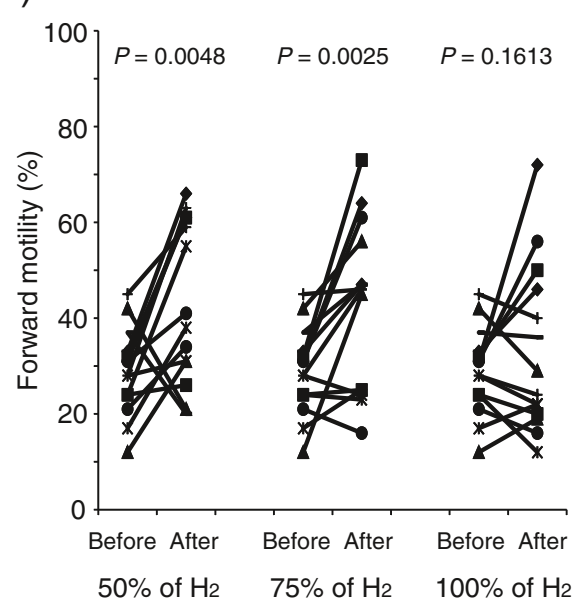

Figure 6 Effect of $\mathbf{H}_{2}$ treatment on human frozen-thawed sperm motility in each suspension. Total frozen-thawed sperm suspensions ( $n=21$ ) were divided into 2 groups, those with higher (forward motility $\geq 50 \%$, normozoospermic, $n=6)$ or lower $(<50 \%$, asthenozoospermic, $\mathrm{n}=15$ ) motility. Sperm motility was determined before and after treatment with sperm-wash medium containing $50 \%$, $75 \%$ and $100 \%$ of $\mathrm{H}_{2}$ for $30 \mathrm{~min}$. Data represent means $\pm \mathrm{SD}$. ${ }^{*} P<0.05$, versus the untreated sperm suspension (a). Comparison of forward motility before and after treatment of each sperm suspension with higher (b) or lower (c) motility. P-values were determined using paired $t$-test.

of highly damaged sperms was more sensitive to easily assess the effect of $\mathrm{H}_{2}$ on sperm motility.

The rate of forward motility increased from 3.6\% to $16.8 \%$ (Figure 1a). The change in motility by $\mathrm{H}_{2}$ treatment was higher than or similar to results previously reported, e.g., $17.95 \%$ to $25.1 \%$ for treatment with platelet-activating factor [27] and a $21 \%$ increase for exogenous pyruvate treatment [28]. Because the increase of forward motility was observed within only $30 \mathrm{~min}, \mathrm{H}_{2}$ treatment may stimulate cell signaling and activate mitochondria, but not transcription and translation in sperm. However, it remains to be elucidated whether the stimulatory effects are dependent on a property of $\mathrm{H}_{2}$ as a reductant. Furthermore, $\mathrm{H}_{2}$, but not $\mathrm{N}_{2}$, stimulated sperm motility, indicating that the effect of $\mathrm{H}_{2}$ did not rely on mechanical stimuli.

Effects were evident in almost all sperm suspensions (16 out of 17) with higher survival rates in which an increase of forward motility was observed with $\mathrm{H}_{2}$ treatment (Figure 2). Slight increase of the motility treated with $\mathrm{N}_{2}$ may due to the effect of mixing and shaking of sperm suspension during experimental manipulation [29]. The samples used in this study were collected from various patients with different physiological conditions and genetic backgrounds; therefore, it is possible that the effects of $\mathrm{H}_{2}$ rely on the character of each sperm suspension. The lack of response in forward motility in sperm suspensions with low survival rates indicated that $\mathrm{H}_{2}$ treatment was less effective on complete or nearly complete necrozoospermia. However, we found that $\mathrm{H}_{2}$ treatment stimulated a very sluggish motility of several sperms in 3 out of 13 suspensions with low survival rates, further indicating that $\mathrm{H}_{2}$ is beneficially effective on movement of sperms with both high and low survival rates. The differential effects of $\mathrm{H}_{2}$ treatment may be dependent on various conditions of sperm. 
$\mathrm{H}_{2}$ did not enhance the sperm swimming speed (Figure 3), indicating that $\mathrm{H}_{2}$ did not hyperactivate sperm. Hyperactivation is characterized by a more energetic and less symmetric beat of sperm flagella and can be achieved in vitro by seminal plasma removal and incubation of sperm in capacitating medium. However, several reagents, including caffeine, which can stimulate sperm movement, have no effect on sperm velocity [30]. On the other hand, exogenous pyruvate accelerates glycolysis, and stimulates motility and hyperactivation with an increase in intracellular ATP levels [28]. It has been reported that glucose-derived ATP during capacitation involves hyperactivation [31], indicating that $\mathrm{H}_{2}$ treatment does not affect glycolysis.

We found that the higher sperm motility induced by $\mathrm{H}_{2}$ treatment was maintained for $2.5 \mathrm{~h}$, even in the absence of $\mathrm{H}_{2}$ (Figure 4), which is clinically enough time for IVF and ICSI. The decrease in motility after $\mathrm{H}_{2}$ treatment is due to several reasons, including the reduction of intracellular ATP, and oxidative stress of oxygen in the room air [32]. Nevertheless, the motility increased the following day after a second $\mathrm{H}_{2}$ treatment. The increase of motility with second treatment of $\mathrm{H}_{2}$ was still higher than that of $\mathrm{N}_{2}$, indicating that the repeated increase was due to $\mathrm{H}_{2}$, but not mechanical stimuli. The repeated stimulation of sperm is likely to be useful in a clinical setting. Higher variation of the mobility in $\mathrm{H}_{2}$ treated sperm in Figure 4 may be dependent on its higher mobility, because the difference of coefficients of variation between the mobility of $\mathrm{H}_{2}$-treated sperm $(0.26$ to $0.59 \%)$ and that of $\mathrm{N}_{2}(0.48$ to $0.98 \%)$ was very low.

A functional relationship among sperm mitochondrial membrane potential, sperm motility, and fertility potential has been proposed [33]. We found that $\mathrm{H}_{2}$ treatment enhanced mitochondrial membrane potential (Figure 5). Since sperm motility is dependent on ATP content, we speculate that $\mathrm{H}_{2}$ treatment enhances mitochondrial function, promotes ATP production, and then stimulates sperm motility. Indeed, we observed previously that $\mathrm{H}_{2}$ prevented a decrease in cellular levels of ATP synthesized in mitochondria [14]. Precise measurements of both ATP and calcium in sperm are needed before and after treatment with $\mathrm{H}_{2}$.

Finally, we used frozen-thawed sperm suspensions to validate the effects of $\mathrm{H}_{2}$ treatment on the motility of damaged sperm. Frozen sperm suspension is used routinely in assisted reproduction treatment. However, freezing has been reported to cause changes in sperm morphology, including damage to mitochondria. Sperm motility is particularly sensitive to freezing damage [34,35]. Effects were evident in 13 out of 15 suspensions of low motile frozenthawed sperm $(86.7 \%)$ in which an increase of forward motility was observed with sperm-wash medium containing $50 \%$ of $\mathrm{H}_{2}$ (Figure $6 \mathrm{~b}$ ), indicating that $\mathrm{H}_{2}$ treatment is clinically a potential approach to activate low motility sperm. However, treatment with medium containing higher concentration, $100 \%$, of $\mathrm{H}_{2}$ was not effective, indicating the possibility that very low concentration of $\mathrm{O}_{2}$ in the medium might repress the sperm motility [36]. Further study is needed to elucidate a dose-dependency of $\mathrm{H}_{2}$-treatment.

The fertilization rates after IVF and ICSI using normal sperm are approximately $60 \%$ and $70 \%$, respectively [37]. In general, the decision to perform either IVF or ICSI is dependent on sperm quality [38], which is determined by the total number of motile sperm [39]. While the concentration and morphology of ejaculated sperm do not affect ICSI results, the injection of a completely immotile spermatozoon is likely to have a negative effect on fertilization and the pregnancy rate $[21,40]$. Currently, there is no efficient therapy for asthenozoospermia.

\section{Conclusion}

The findings of this study strongly indicate that $\mathrm{H}_{2}$ treatment activates low motility sperms. Importantly, recent studies demonstrated that $\mathrm{H}_{2}$ might have potential for wide use in medical applications as a novel, safe, effective antioxidant with minimal side effects [14-19]. We propose here that $\mathrm{H}_{2}$ is a new promising agent for male infertility treatment. However, to make practical use of $\mathrm{H}_{2}$ treatment, we further need to examine the effects of $\mathrm{H}_{2}$-treated sperm on fertilizing ability, embryonic development and safety for IVF and ICSI in future studies.

\section{Abbreviations \\ Cl: Confidence interval; ICSI: Intracytoplasmic sperm injection; IVF: In vitro fertilization; MTG: MitoTracker Green; PPF: Plasma protein fraction; ROS: Reactive oxygen species; SD: Standard deviation; \\ TMRM: Tetramethylrhodamine methyl ester.}

\section{Competing interests}

The authors declare that they have no competing interests.

\section{Authors' contributions}

$\mathrm{KN}$ involved in conceiving and designing the study, analyzing and interpreting the data; NY in provision of materials, patients and resources; YN in technical support; $1 O$ in organization of this study and writing the article. All authors read and approved the final manuscript.

\section{Acknowledgments}

The work was funded by research funding (KAKENHI 30343586) from the Ministry of Education, Culture, Sports, Science and Technology (MEXT) of Japan. We are greatful to Naoki Nakayama, Kayoko Ikegami, Aya Nakanishi, Mutsumi Abe, and Hitomi Watanabe (Yamashita Shonan Yume Clinic) for collection of samples.

\section{Author details}

${ }^{1}$ Biological Process of Aging, Tokyo Metropolitan Institute of Gerontology, 35-2 Sakae-cho, Itabashi-ku, Tokyo 173-0015, Japan. ${ }^{2}$ Reproductive Medicine Research Center, Yamashita Shonan Yume Clinic, 1-2-10 Kugenumaishigami, Fujisawa 251-0025, Japan. ${ }^{3}$ Animal Facility, Tokyo Metropolitan Institute of Gerontology, 35-2 Sakae-cho, Itabashi-ku, Tokyo 173-0015, Japan.

Received: 28 November 2014 Accepted: 27 December 2014

Published online: 10 January 2015 


\section{References}

1. Matzuk MM, Lamb DJ. The biology of infertility: research advances and clinical challenges. Nat Med. 2008;14:1197-213.

2. Dhillon VS, Shahid M, Husain SA. Associations of MTHFR DNMT3b 4977 bp deletion in mtDNA and GSTM1 deletion, and aberrant CpG island hypermethylation of GSTM1 in non-obstructive infertility in Indian men. Mol Hum Reprod. 2007;13:213-22.

3. Ruiz-Pesini E, Lapeña AC, Díez-Sánchez C, Pérez-Martos A, Montoya J, Alvarez E, et al. Human mtDNA haplogroups associated with high or reduced spermatozoa motility. Am J Hum Genet. 2000;67:682-96.

4. Torra R, Sarquella J, Calabia J, Martí J, Ars E, Fernández-Llama P, et al. Prevalence of cysts in seminal tract and abnormal semen parameters in patients with autosomal dominant polycystic kidney disease. Clin J Am Soc Nephrol. 2008;3:790-3.

5. Gharagozloo P, Aitken RJ. The role of sperm oxidative stress in male infertility and the significance of oral antioxidant therapy. Hum Reprod. 2011;26:1628-40

6. Delbarco-Trillo J, Roldan ER. Effects of metabolic rate and sperm competition on the fatty-acid composition of mammalian sperm. J Evol Biol. 2013. doi:10.1111/jeb.12275.

7. El-Taieb MA, Herwig R, Nada EA, Greilberger J, Marberger M. Oxidative stress and epididymal sperm transport, motility and morphological defects. Eur J Obstet Gynecol Reprod Biol. 2009;144 Suppl 1:S199-203.

8. Sousa MI, Amaral S, Tavares RS, Paiva C, Ramalho-Santos J. Concentrationdependent Sildenafil citrate (Viagra) effects on ROS production, energy status, and human sperm function. Syst Biol Reprod Med. 2014;60:72-9.

9. Eskenazi B, Kidd SA, Marks AR, Sloter E, Block G, Wyrobek AJ. Antioxidant intake is associated with semen quality in healthy men. Hum Reprod. 2005:20:1006-12.

10. Schmid TE, Eskenazi B, Marchetti F, Young S, Weldon RH, Baumgartner A, et al. Micronutrients intake is associated with improved sperm DNA quality in older men. Fertil Steril. 2012;98:1130-7.

11. Talevi R, Barbato V, Fiorentino I, Braun S, Longobardi S, Gualtieri R. Protective effects of in vitro treatment with zinc, $d$-aspartate and coenzyme q10 on human sperm motility, lipid peroxidation and DNA fragmentation. Reprod Biol Endocrinol. 2013;11:81.

12. Condorelli RA, La Vignera S, Bellanca S, Vicari E, Calogero AE. Myoinositol: does it improve sperm mitochondrial function and sperm motility? Urology. 2012;79:1290-5.

13. Ebner T, Tews G, Mayer RB, Ziehr S, Arzt W, Costamoling W, et al. Pharmacological stimulation of sperm motility in frozen and thawed testicular sperm using the dimethylxanthine theophylline. Fertil Steril. 2011;96:1331-6

14. Ohsawa I, Ishikawa M, Takahashi K, Watanabe M, Nishimaki K, Yamagata K, et al. Hydrogen acts as a therapeutic antioxidant by selectively reducing cytotoxic oxygen radicals. Nat Med. 2007;13:688-94.

15. Hayashida K, Sano M, Ohsawa I, Shinmura K, Tamaki K, Kimura K, et al. Inhalation of hydrogen gas reduces infarct size in the rat model of myocardial ischemia-reperfusion injury. Biochem Biophys Res Commun. 2008;373:30-5

16. Fukuda K, Asoh S, Ishikawa M, Yamamoto Y, Ohsawa I, Ohta S. Inhalation of hydrogen gas suppresses hepatic injury caused by ischemia/reperfusion through reducing oxidative stress. Biochem Biophys Res Commun 2007;361:670-4.

17. Oharazawa H, Igarashi T, Yokota T, Fujii H, Suzuki H, Machide M, et al. Protection of the retina by rapid diffusion of hydrogen: administration of hydrogen-loaded eye drops in retinal ischemia-reperfusion injury. Invest Ophthalmol Vis Sci. 2010;51:487-92.

18. Cardinal JS, Zhan J, Wang Y, Sugimoto R, Tsung A, McCurry KR, et al. Oral hydrogen water prevents chronic allograft nephropathy in rats. Kidney Int. 2010;77:101-9.

19. Terasaki Y, Ohsawa I, Terasaki M, Takahashi M, Kunugi S, Dedong K, et al. Hydrogen therapy attenuates irradiation-induced lung damage by reducing oxidative stress. Am J Physiol Lung Cell Mol Physiol. 2011;301:L415-26.

20. World Health Organization. WHO laboratory manual for the examination and processing of human semen. 5th ed. Geneva: WHO Press; 2010.

21. Liu J, Nagy Z, Joris H, Tournaye H, Devroey P, Van Steirteghem AC. Intracytoplasmic sperm injection does not require special treatment of the spermatozoa. Hum Reprod. 1994;9:1127-30.

22. Makler A. The improved 10 mic. chamber for rapid sperm count and motility evaluation. Fertil Steril. 1980;33:337-8.
23. ESHRE Andrology Special Interest Group. Guidelines on the application of CASA technology in the analysis of spermatozoa. Hum Reprod. 1998;13:142-5.

24. Zavos PM, Sofikitis N, Toda T, Miyagawa I. Improvements in qualitative characteristics of cryopreserved human spermatozoa following recovery via the SpermPrep II filtration method. Tohoku J Exp Med. 1991;165:283-90.

25. Marchetti C, Jouy N, Leroy-Martin B, Defossez A, Formstecher P, Marchetti P. Comparison of four fluorochromes for the detection of the inner mitochondrial membrane potential in human spermatozoa and their correlation with sperm motility. Hum Reprod. 2004;19:2267-76.

26. Angelopoulou R, Lavranos G, Manolakou P. ROS in the aging male: model diseases with ROS-related pathophysiology. Reprod Toxicol. 2009;28:167-71.

27. Esmaeilpour T, Zarei MR, Bahmanpour S, Aliabadi E, Hosseini A, Jaberipour M. Effect of follicular fluid and platelet-activating factor on lactate dehydrogenase C expression in human asthenozoospermic samples. Iran J Med Sci. 2014;39:20-8.

28. Hereng TH, Elgstøen KB, Cederkvist FH, Eide L, Jahnsen T, Skålhegg BS, et al. Exogenous pyruvate accelerates glycolysis and promotes capacitation in human spermatozoa. Hum Reprod. 2011;26:3249-63.

29. Makler A, Jakobi P. Effects of shaking and centrifugation on human sperm motility. Arch Androl. 1981;7:21-6.

30. Moussa MM. Caffeine and sperm motility. Fertil Steril. 1983;39:845-8.

31. Williams AC, Ford WC. The role of glucose in supporting motility and capacitation in human spermatozoa. J Androl. 2001:22:680-95.

32. Luconi M, Forti G, Baldi E. Pathophysiology of sperm motility. Front Biosci. 2006;11:1433-47

33. Kasai T, Ogawa K, Mizuno K, Nagai S, Uchida Y, Ohta S, et al. Relationship between sperm mitochondrial membrane potential, sperm motility, and fertility potential. Asian J Androl. 2002;4:97-103.

34. O'Connell M, McClure N, Lewis SE. The effects of cryopreservation on sperm morphology, motility and mitochondrial function. Hum Reprod. 2002;17:704-9.

35. Boitrelle F, Albert M, Theillac C, Ferfouri F, Bergere M, Vialard F, et al. Cryopreservation of human spermatozoa decreases the number of motile normal spermatozoa, induces nuclear vacuolization and chromatin decondensation. J Androl. 2012;33:1371-8.

36. NEVO AC. Dependence of sperm motility and respiration on oxygen concentration. J Reprod Fertil. 1965;9:103-7.

37. Check JH, Bollendorf A, Wilson C, Summers-Chase D, Horwath D, Yuan W. A retrospective comparison of pregnancy outcome following conventional oocyte insemination vs intracytoplasmic sperm injection for isolated abnormalities in sperm morphology using strict criteria. J Androl. 2007;28:607-12.

38. Vellani E, Colasante A, Mamazza L, Minasi MG, Greco E, Bevilacqua A. Association of state and trait anxiety to semen quality of in vitro fertilization patients: a controlled study. Fertil Steril. 2013;99:1565-72.

39. Stalf T, Mehnert C, Hajimohammad A, Manolopoulos K, Shen Y, Schuppe $\mathrm{HC}$, et al. Influence of motility and vitality in intracytoplasmic sperm injection with ejaculated and testicular sperm. Andrologia. 2005;37:125-30.

40. Liu J, Nagy Z, Joris H, Tournaye H, Smitz J, Camus M, et al. Analysis of 76 total fertilization failure cycles out of 2732 intracytoplasmic sperm injection cycles. Hum Reprod. 1995;10:2630-6.

\section{Submit your next manuscript to BioMed Central and take full advantage of:}

- Convenient online submission

- Thorough peer review

- No space constraints or color figure charges

- Immediate publication on acceptance

- Inclusion in PubMed, CAS, Scopus and Google Scholar

- Research which is freely available for redistribution 